\title{
The application of optical methods for the assessment of the aesthetic compatibility of architectural concrete
}

\author{
Tomasz Benedysiuk ${ }^{1 *}$, and Wioletta Jackiewicz-Rek ${ }^{1}$ \\ ${ }^{1}$ Warsaw University of Technology, Faculty of Civil Engineering, Poland
}

\begin{abstract}
Architectural concrete is usually defined as concrete for with the requirements regarding aesthetics of its surface are formulated. The currently applied requirements regarding aesthetics compatibility are mostly based on subjective methods of concrete surface assessment. While it is relatively easy to apply quantitative criteria for components of surface analysis such as porosity, the quantitative assessment of colour compatibility could be problematic. In this paper, an optical method for the assessment of concrete surface colour is presented. The main goal of the investigation was to assess the effect of modifications of the aggregate used for preparation of architectural concrete mix on the surface colour. Optical test methods using a self-made DARK-BOX device were used. The obtained results confirmed the usefulness of the method used to assess the colour compatibility of architectural concrete surfaces.
\end{abstract}

\section{Introduction}

Architectural concrete can be defined in various ways. One definition is as follows: 'visible concrete surface that is not covered by an opaque coating'. The formwork sheathing, the concrete mix design, the type of concrete care and the surface treatment enable any surface shaping and texture of concrete [1]. As architectural concrete is a type of concrete that is the finishing element, we should use different criteria for its assessment than for other concrete surfaces. One of the features that should distinguish architectural concrete is the aesthetic compatibility of the concrete surface. Aesthetic compatibility can be defined as the set of quantified surface features of an element that are responsible for the positive reception of the surface with regard to aesthetics [2]. The following features of smooth architectural concrete surface that affect its aesthetic reception, can be included: porosity, colour properties, gloss.

The currently applied requirements regarding aesthetics compatibility are mostly based on subjective methods of concrete surface assessment. While it is relatively easy to apply quantitative criteria for components of surface analysis such as porosity, the quantitative assessment of colour compatibility could be problematic. In this paper, concrete surface colour assessment with an optical method is presented. The main goal of the investigation

\footnotetext{
* Corresponding author: tomasz.benedysiuk@gmail.com
} 
was to assess the effect of modifications of aggregate used for preparation of the architectural concrete mix on the colour of the surface.

\section{Factors affecting surface perception}

There are many factors affecting colour that reach the receptor - whether it be the human eye or the camera image sensor. The receptor interprets which waves reach it and transforms this information into a colour impression.

The first factor that affects colour perception is the light source. Light emitted by the source is partly absorbed and partly reflected by the object. Part of the light can also pass through the object (transmission). The reflection coefficient (reflectance) depends, inter alia, on the wavelength of the incident on the object. Depending on the lighting, the way we perceive the colour of the object may change.

The physicochemical properties of the material are another factor affecting the reflection coefficient of a given wavelength. In particular, materials with different chemical compositions may produce identical visual impressions under certain lighting, but visual impressions may change with the change of light source type. This phenomenon is called metamerism.

Another important feature of the surface that affects colour impression is the angle at which we observe the object. When the angle between the light source, the observed surface point and the observer changes, a different amount of reflected light reaches the recipient. Therefore, to measure colours with spectrophotometers, measurement geometries should be defined. For example, it could be $45 / 0$ geometry or a spherical geometry (d/8). The specificity of any concrete surface is its unevenness in colour. This is because in the process of cement hydration, even a small difference in the amount of mixing water at a given point has a big impact on the final colour [3].

Fig. 1. Unevenness in surface coloration based on the example of a $10 \times 10 \mathrm{~cm}$ section of concrete surface.

\section{Methods of colour measurement}

One of the popular methods of colour measurement is to measure the colour with a colorimeter or spectrophotometer. Such devices use a CIE standardised light source, e.g. D65. These devices examine the colour of the area from a few to several square millimeters 
with the determined measurement geometry. Due to the above-mentioned colour unevenness in the concrete surface, the assessment of colour with such devices is inadequate as determining a reliable measurement of the average colour of an element would require multiple measurements of individual points, and even this would not guarantee that the obtained result would be reliable.

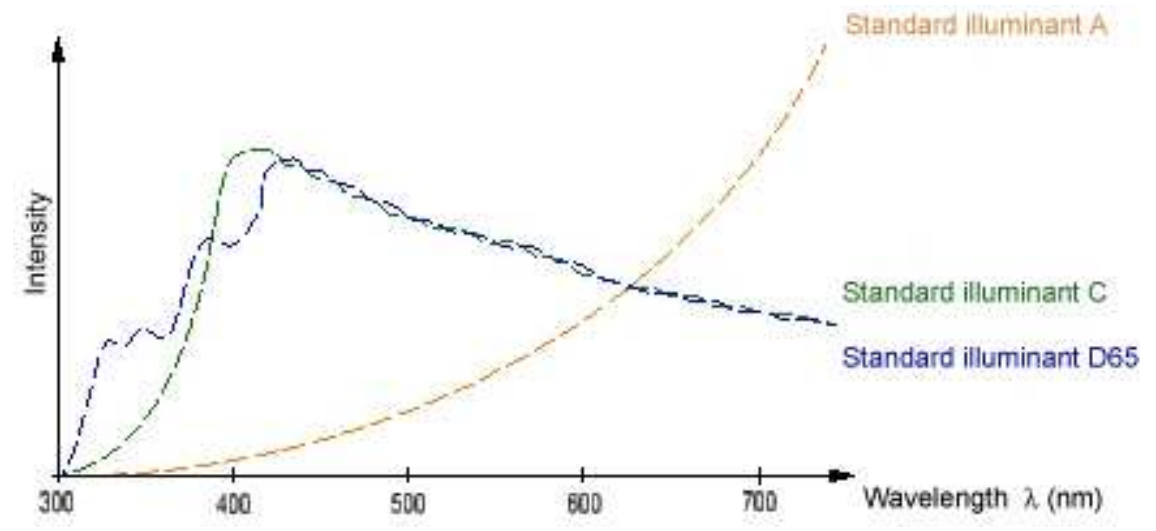

Fig. 2. Graph showing spectra of CIE standardised light sources.

https://www.sensorinstruments.de/whatiswhat.php?subpage=11\&language=pl

Another method of colour measurement presented by Sönke Johnsen [4] uses calibrated photographs. Primarily, it requires identical lighting conditions for each measured surface fragment and for the calibration pattern.

Another option is to collect an appropriate number of sample surfaces to photograph. Then, the sample surfaces must be divided into classes. Also, the classifier that assigns the measured surface to a given class must be made. One of the types of classification methods can be determining whether a given surface meets the criteria of similarity with a given reference surface. Such techniques are currently used for the acceptance of architectural concrete, but without applying strict quantitative criteria when classifying surfaces [1]. Using this classification, it is necessary to ensure the repeatability of the conditions in which the surfaces are photographed - this applies to both lighting conditions and camera settings. However, there is no need to provide identical lighting conditions on every point of the surface or to provide a light source with a spectrum identical to sunlight.

\section{Materials and methods}

The aim of the study was to develop a classifier that would compare two surfaces of architectural concrete in order to assess the effect of the aggregate modification used in the production of the concrete mix on the colour of the surface.

The subjects of the study were two architectural concrete slabs with dimensions of around $30 \times 30 \mathrm{~cm}$, both were made using white cement. The only difference was the type of aggregate used - in slab No. 1 it was Vistula sand, in slab No. 2 white sand was used.A slab of non-architectural concrete was added to study for comparison. The surface of a white sheet of paper which was the reference element was also examined.

A DARK-BOX device constructed by the author of this work was used for the research. It consists of a rectangular box painted from the inside in black, open from the bottom side. At the top from the inner side there are mounted LED SMD 2835 lights, and in the central part there is a hole for the lens of a digital camera. Such a device does not provide homogeneous lighting conditions of the entire sample surface, and the light spectrum of the 
used LED lamps does not match the spectrum of sunlight. However, it provides constant, repeatable lighting conditions, which is sufficient to use the device for concrete surface classification. This research approach results from the relationships presented in Section 3.

Due to the previously described unevenness of concrete surface colour, the adopted test method also had to meet the criteria of the simultaneous assessment of a surface area that was as large as possible. It should be noted that in this case, each section of the surface is illuminated at a different angle. The adopted method of classification selection does not require illuminating each part of surface from the same angle.

The choice was also dictated by the fact that the DARK-BOX device also provides the opportunity to examine the porosity and other features of the concrete surface. This allows the enclosure of all the equipment needed to examine the features of the concrete surface for aesthetic compatibility in one device.

The proposed methodology is based on previous studies [7] in which $\mathrm{x}$ surfaces of architectural and non-architectural concrete were studied. Additionally, the testing device has been redesigned to provide better testing conditions.

It was decided to use a research method that compares the classified surfaces to the reference element. As the idea of the creators of the tested panels was to make the surfaces of the element as white as possible, the surface of white paper was used as the reference element.

The study consisted of taking images of samples in the same, homogeneous lighting conditions and then determining the parameters (RGB components) of 9 adjacent $4.5 \times 4.5$ $\mathrm{cm}$ fields on each surface - the field size was selected to eliminate slight surface imperfections that were not the subject of the study. The average value was then calculated for each RGB component. The distance D of the tested surface from the pattern was then determined using the formula (4).

$$
\begin{gathered}
\Delta R=\left(R_{W}-R_{P}\right) \\
\Delta G=\frac{\left(G_{W}-G_{P}\right) \cdot R_{B}}{G_{B}} \\
\Delta B=\frac{\left(B_{W}-B_{P}\right) \cdot R_{B}}{E_{B}} \\
D=\sqrt{3 \cdot \Delta R^{2}+4 \cdot \Delta G^{2}+2 \cdot \Delta B^{2}}
\end{gathered}
$$

where:

$\mathrm{R}_{\mathrm{W}}$ - value $\mathrm{R}$ of reference surface

$\mathrm{G}_{\mathrm{W}}$ - value $\mathrm{G}$ of reference surface

$B_{W}$ - value $B$ of reference surface

$R_{P}$ - value $R$ of tested surface

$G_{P}$ - value $G$ of tested surface
$B_{P}$ - value $B$ of tested surface

$R_{B}$ - value $R$ of achromatic reference surface

$G_{B}$ - value $G$ of achromatic reference surface

$B_{B}$ - value $B$ of achromatic reference surface

Formulas (1-3) have been proposed in such a way as to minimise the effect of white balance selection and are used to calculate the relative difference of a given component between the reference surface and the test surface. The D parameter is the weighted Euclidean distance between the tested surface and the reference surface. The proposed formulas are a compilation of experiences from previous research and the methods used to compare colours with each other [8].

In the studied case, the standard was equal to the achromatic reference surface. It should be noted that the proposed method may not fully reflect the visual experience of a human 
observing a given surface, but it is an attempt to quantify the tested surfaces with defined criteria and a testing device.

\section{Results and discussion}

Pictures taken with the DARK-BOX device are shown in Fig. 3. The greenish colouring of the photographs was caused by parameters of the white balance, which, however, was set identically for each of the photographs - thus, identical test conditions for each surface were ensured. In addition, patterns (1-3) were constructed to maximally reduce the effect of white balance selection. The obtained results (Table 1) show that sample No. 1 with the Vistula sand more closely matches the reference element than sample No. 2. Pictures taken with the DARK-BOX device are shown in Fig. 3.
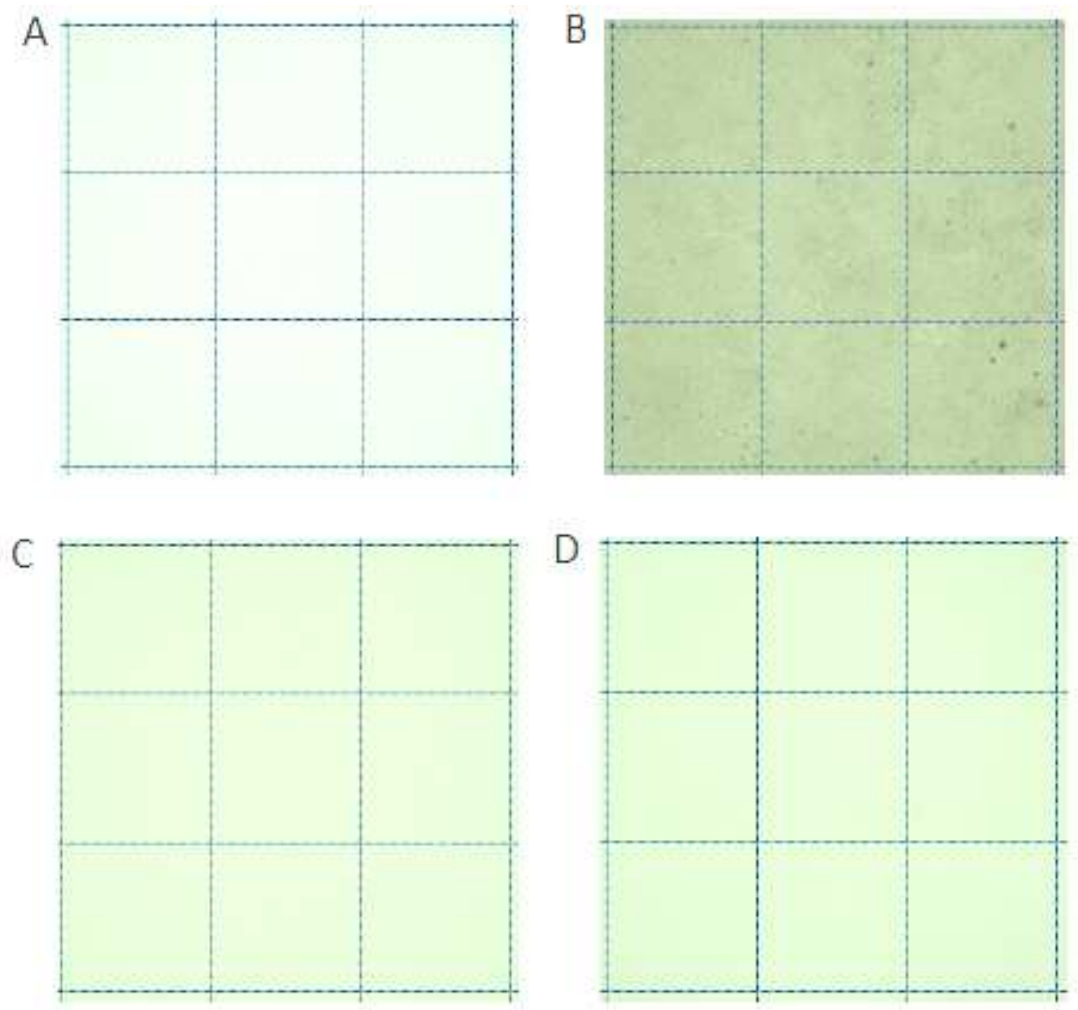

Fig. 3. Photographs of tested surfaces A. white sheet, B. plain non-architectural concrete, C. Surface No. 1 - concrete with Vistula sand, D. Surface No. 2 - concrete with white sand. 
Table 1. Classification results of tested surfaces.

\begin{tabular}{|c|c|c|c|c|}
\hline Parameter name & Ref. elem. & Concrete & Sample No. 1 & Sample No. 2 \\
\hline mean R (0-255) & 191.22 & 139.78 & 181.00 & 176.56 \\
\hline mean G (0-255) & 215.33 & 158.67 & 206.11 & 201.33 \\
\hline mean B (0-255) & 189.89 & 115.22 & 166.89 & 162.78 \\
\hline stand. deviation R & 2.22 & 1.86 & 1.80 & 1.81 \\
\hline stand. deviation G & 1.73 & 2.12 & 1.62 & 2.00 \\
\hline Std Dev' B & 1.90 & 1.72 & 1.27 & 1.64 \\
\hline relative difference R & 7.00 & 6.00 & 6.00 & 5.00 \\
\hline relative difference G & 5.00 & 6.00 & 5.00 & 6.00 \\
\hline relative difference B & 6.00 & 5.00 & 4.00 & 5.00 \\
\hline Distance (D) & 0.00 & 171.39 & 40.68 & 52.48 \\
\hline
\end{tabular}

The values of standard deviation were very low, which shows that the level of illumination was close to uniform. Moreover, low standard deviations and similar values of relative differences indicate that the size of the studied area was well chosen as the visible point imperfections of non-architectural concrete probably do not affect the measurement result. It is also interesting that the standard deviation for individual parameters of the reference element, despite being similar for all surfaces, is not generally smaller than for the other surfaces. This is probably due to the slight difference in gloss of the individual surfaces.

The question that may arise during analysis of the results is whether sample No. 2, despite having obtained a worse result than sample No. 1, can be classified as sufficiently similar to sample No. 1. To answer this question, it was decided to compare sample No. 2 to sample No. 1 using the above formulas (1-4). The results of the comparison are shown in Table 2.

Table 2. Calculated difference between Sample No. 1 and Sample No. 2.

\begin{tabular}{|c|c|c|}
\hline Parameter name & Sample No. 1 & Sample No. 2 \\
\hline mean R (0-255) & 180.77 & 176.52 \\
\hline mean G (0-255)) & 205.70 & 201.45 \\
\hline mean B (0-255) & 166.60 & 163.20 \\
\hline Distance (D) & 0.00 & 11.60 \\
\hline
\end{tabular}

The obtained result shown in Table 2 and the comparison of the results from Table 1 indicate a small relative difference between Sample No. 1 and Sample No. 2. The question arises of whether it is a sufficient value to consider that these samples are sufficiently similar. The answer to such a problem may be problematic due to the imprecision of the word "sufficient". Nevertheless, it would be possible to conduct a study comparing surfaces 
using a survey and the measurement technique presented above and draw conclusions on this basis.

\section{Conclusions}

The study showed that the proposed method, as well as developed DARKBOX optical device, can be used to assess the aesthetic compatibility of the surfaces of architectural concretes. The tests performed with the DARKBOX device confirmed that the substitution of white sand with Vistula river sand did not significantly change the aesthetic properties of the concrete surface. However, additional research should be carried out to determine how closely the classification formula corresponds to the human visual experience.

The test result is affected not only by the colour of the surface but also to some extent by its gloss. Using the proposed research method, a surface catalogue could be created. The tested surfaces could then be classified with regard to their closeness to a specific reference surface.

\section{References}

1. W. Jackiewicz-Rek, P. Woyciechowski, Materiały Budowlane 2, 20-21 (2014) (in Polish)

2. B. Bissonnette, L. Courard, A. Garbacz, Concrete Surface Engineering (151-152, 2016)

3. E. Szymczak, M. Oleksik, Budownictwo, Technologie, Architektura, 2, 58-61 (2018) (in Polish)

4. S. Johnsen, J. Exp. Biol. 219, 772-778 (2016)

5. G. Sharma, Digital Color Imaging Handbook (1.7.2 ed., 2003)

6. G. Klinker, S. Shafer, T. Kanade, Proceedings of the First International Conference on Computer Vision (145-150, 1987)

7. T. Benedysiuk, Nowa, ilościowa metoda oceny jakości powierzchni gładkiego betonu architektonicznego [Unpublished masters's thesis] (Warsaw University of Technology, 2016) (in Polish)

8. A. Gijsenij, T. Gevers, M.P. Lucassen, ECCV LNCS, 5302, 145-150 (2008)

9. D. Bennett, Architectural Institute of Concrete (2007)

10. E. G. Nawy, Concrete Construction Engineering Handbook (2008) 